\title{
Supporting Information \\ Generalization of Langevin Dynamics from Spatio-Temporal Dressed Dynamics Perspective
}

\author{
Kaicheng Zhu and Haibin Su* \\ Department of Chemistry, The Hong Kong University of Science and Technology, \\ Clear Water Bay, Kowloon, Hong Kong
}

*Corresponding Author:

E-mail: haibinsu@ust.hk

1. The interaction between particle and medium model

2. Spatio-temporal kernel using Green's function in dressed dynamics

3. The local-coupling limit of dressed dynamics equation

4. An aqueous solvation shell model

5. Confinement effect on dressed dynamics of an aqueous solvation shell

6. Time resolved viscosity of aqueous solvation shell system

Figure S1. The radius dependence of the lifetime distribution of aqueous solvation shells at different temperatures.

Figure S2. The time dependent MSD of the particle for the system with $l$ of $2 \mathrm{~nm}$.

Figure S3. The VAC function of particle for the system with $l$ of $1 \mathrm{um}$.

Figure S4. The VAC function of the particle for the system with $l$ of $2 \mathrm{~nm}$.

Figure S5. The time-dependent dynamic exponent of the dressed dynamics of aqueous solvation shells. 


\section{The interaction between particle and medium model}

In a system where the total number of particles is constant as $N$ and every particle could be identified with time-independent mass, the dynamics of particle in 3 dimensional space follows the principle of classical mechanics:

$$
m_{i} \frac{d^{2} \boldsymbol{y}_{i}}{d t^{2}}=\boldsymbol{F}_{i}(\boldsymbol{y}, t)=\sum_{j=1, j \neq i}^{N} \boldsymbol{F}_{i j}\left(\boldsymbol{y}_{i}, \boldsymbol{y}_{j}, t\right)
$$

where $m_{i}$ is the mass of the $i$-th particle, $\boldsymbol{y}_{i}$ is the spatial position vector of the $i$-th particle, $\boldsymbol{F}_{i}$ is the net force acting on it, $\boldsymbol{y}$ is the $N \times 3$ system state matrix composed of every $\boldsymbol{y}_{i}$ for all the $N$ particles as the systematic characterization of the complete dynamics, and $\boldsymbol{F}_{i j}$ is the timedependent force operator that produces the interaction between the $i$-th and the $j$-th particle based on their individual state. The time dependence of $\boldsymbol{F}_{i}$ and $\boldsymbol{F}_{i j}$ shows the non-conservative nature of a general non-isolated system, because of the entropy production and the energy exchange through the thermostat. In an isolated deterministic system, the total energy is conserved, so the net force reduces to the time-independent form as $\boldsymbol{F}_{i}(\boldsymbol{y})$. In the weakly interactive homogeneous equilibrium limit, we assume the state of the system to approach $\overline{\boldsymbol{y}}$, corresponding to a pseudo-equilibrium phase. With this pseudo-equilibrium state as the reference, the collective interaction $\boldsymbol{F}_{i}$ is separated into two distinct parts as follows:

$$
\boldsymbol{F}_{i}(\boldsymbol{y}, t)=\boldsymbol{F}_{i}^{(0)}\left(\boldsymbol{y}_{i}, \overline{\boldsymbol{y}}, t\right)+\sum_{j=1, j \neq i}^{N} \boldsymbol{F}_{i j}^{(1)}\left(\boldsymbol{y}_{i}, \boldsymbol{y}_{j}-\overline{\boldsymbol{y}}_{j}, t\right)
$$

where $\boldsymbol{F}_{i}^{(0)}\left(\boldsymbol{y}_{i}, \overline{\boldsymbol{y}}, t\right)$ is the force associated with near-equilibrium fluctuation and dissipation on the $i$-th particle, and $\boldsymbol{F}_{i j}^{(1)}$ are the other force components describing the contribution of farfrom-equilibrium interactions. $\boldsymbol{F}_{i}^{(0)}$ results from the near-equilibrium interaction between the $i$-th particle and the homogeneous equilibrium reference state of the medium, so it can be expressed by the similar manner as in the linear response theory.

The interaction in the first term on the right-hand side (RHS) in Eq. S2 is described by the dissipation and fluctuation forces in the Langevin equation, which characterizes the interaction from an equilibrium medium toward a Brownian particle, the same as the physical nature of $\boldsymbol{F}_{i}^{(0)}$ :

$$
\boldsymbol{F}_{i}^{(0)}\left(\boldsymbol{y}_{i}, \overline{\boldsymbol{y}}, t\right)=-m_{i} \gamma_{i} \frac{d \boldsymbol{y}_{i}}{d t}+m_{i} \boldsymbol{R}_{i}(t)
$$

where $\gamma_{i}$ is the individual friction constant associated with the velocity of the $i$-th particle and $\boldsymbol{R}_{i}(t)$ is the mass-normalized intrinsic fluctuation force. For the second term on the RHS in Eq. $\mathrm{S} 2$, we define a dynamic vector field $\phi(\boldsymbol{x}, t)$ that implicitly depends on $\boldsymbol{y}_{j}-\overline{\boldsymbol{y}}_{j}$, and an associated vector operator $\widehat{N}_{\left(\boldsymbol{x}, \boldsymbol{y}_{i}\right)}$ that helps to reproduce the same force as $\sum_{j=1, j \neq i}^{N} \boldsymbol{F}_{i j}^{(1)}$ by spatial integral over the entire domain of the system:

$$
m_{i} \int d \boldsymbol{x} \widehat{N}_{\left(\boldsymbol{x}, \boldsymbol{y}_{i}\right)} \phi(\boldsymbol{x}, t)=\sum_{j=1, j \neq i}^{N} \boldsymbol{F}_{i j}^{(1)}\left(\boldsymbol{y}_{i}, \boldsymbol{y}_{j}-\overline{\boldsymbol{y}}_{j}, t\right)
$$

Although neither the path nor the velocity is deterministic in a statistical dynamic system, there always exist the deterministic mappings between the path and the velocity as long as each particle is distinguishable,

$$
\boldsymbol{y}_{i}(t)=\boldsymbol{Y}_{i}\left(\boldsymbol{v}_{i}\right)=\int_{0}^{t} \boldsymbol{v}_{i}\left(t^{\prime}\right) d t^{\prime}+\boldsymbol{y}_{i}(0)
$$


where $\boldsymbol{v}_{i}$ is the instantaneous velocity of the $i$-th particle, $\boldsymbol{Y}_{i}$ is a mapping from the velocity function to the position function whose inverse mapping operator is $\frac{d}{d t}$ for the $i$-th particle, and $\boldsymbol{y}_{i}(0)$ is a time-independent quantity for the initial position of the particle. As a result, $\boldsymbol{y}_{i}$ can be regarded as a function of time mapped from $\boldsymbol{v}_{i}$ by a definite integration in time domain. By this means, we can convert the whole system from path or position state dependent to velocity dependent with all related operators acting on $\boldsymbol{y}_{i}$ changed to acting on $\boldsymbol{v}_{i}$, which results in the coupled particle-medium system described by $\boldsymbol{v}$ and $\phi$.

\section{Spatio-temporal kernel using Green's function in dressed dynamics}

In general, the effective field $\phi(x, t)$ characterizing the collective dynamic properties of the medium could be described by the following equation:

$$
\begin{gathered}
\hat{L}_{\phi} \phi(x, t)=f^{e x}(x, t)+\widehat{M}_{\left(x, y_{i}\right)} \boldsymbol{v}_{i}(t) \\
\hat{B} \phi(x, t)=b(x, t)
\end{gathered}
$$

where $\hat{L}_{\phi}$ is the operator for the intrinsic property of $\phi, \widehat{M}_{x, y_{i}}$ is a vector operator that gives the interaction from the $i$-th particle, $f^{e x}(x, t)$ is an explicit driving function due to external force coming from out of the system, $B$ is the local operator at the spatio-temporal boundary, and $b(\boldsymbol{x}, t)$ denotes the initial and boundary conditions. So, the operators $\widehat{M}_{\boldsymbol{x}, \boldsymbol{y}_{i}}$ and $\widehat{N}_{\boldsymbol{x}, \boldsymbol{y}_{i}}$ together establish the cross-coupling interactions between the particle and the field. Eq. S6b can be absorbed into Eq. S6a with a simple transform.

Here, we define the following transformation to combine the Eq. S7 into one consistent form:

$$
\begin{aligned}
\hat{L}_{\phi}^{\prime} & = \begin{cases}\hat{L}_{\phi} & x, t \in \Omega \\
\hat{B} & x, t \in \partial \Omega\end{cases} \\
f^{\prime} & = \begin{cases}f^{e x} & \boldsymbol{x}, t \in \Omega \\
b & \boldsymbol{x}, t \in \partial \Omega\end{cases} \\
\widehat{M}_{\left(\boldsymbol{x}, y_{i}\right)}^{\prime} & = \begin{cases}\widehat{M}_{\left(x, y_{i}\right)} & \boldsymbol{x}, t \in \Omega \\
\hat{0} & \boldsymbol{x}, t \in \partial \Omega\end{cases}
\end{aligned}
$$

where $\Omega$ is the spatio-temporal domain of the system and $\partial \Omega$ is the associated domain boundary. By this means, Eq. S6a is generalized with the additional initial and boundary conditions absorbed into the new intrinsic operator $\hat{L}_{\phi}^{\prime}$ and the new driving function $f^{\prime}$ while preserving the same mathematical form. With a non-trivial boundary condition, the governing dynamics of $\phi(x, t)$ is thus formulated in an inhomogeneous differential equation system. The detailed linearity of the combined operator $\hat{L}_{\phi}^{\prime}$ depends on both original $\widehat{L}_{\phi}$ and $\hat{B}$, i.e., if both $\widehat{L}_{\phi}$ and $\hat{B}$ are linear, then the transformed operator is also linear. Besides, the new operator $\widehat{M}_{\left(x, y_{i}\right)}^{\prime}$ preserves the original linearity. The corresponding Green's function of this generalized operator $\hat{L}_{\phi}^{\prime}$ thus covers the complete space-time domain of the system including the spatio- 
temporal boundary. If $\hat{L}_{\phi}^{\prime}$ is linear and its corresponding Green's function $\tilde{G}\left(\boldsymbol{x}-\boldsymbol{x}^{\prime}, t-t^{\prime}\right)$ exists, Eq. S6 could be exactly solved across the complete time domain as follows ${ }^{1}$ :

$$
\phi(\boldsymbol{x}, t)=\iint \tilde{G}\left(\boldsymbol{x}-\boldsymbol{x}^{\prime}, t-t^{\prime}\right)\left[f^{\prime}\left(\boldsymbol{x}^{\prime}, t^{\prime}\right)+\widehat{M}_{\left(x^{\prime}, y_{i}^{\prime}\right)}^{\prime} \boldsymbol{v}_{i}\left(t^{\prime}\right)\right] d \boldsymbol{x}^{\prime} d t^{\prime}
$$

If the coupling interactions are well-defined to be state-dependent in a self-consistent way, $\widehat{M}_{\left(x, y_{i}\right)}^{\prime}$ and $\widehat{N}_{\left(x, y_{i}\right)}$ should not have explicit time dependence. Some specific examples of $\widehat{L}_{\phi}^{\prime}$ and $\tilde{G}$ are listed in the Tab. S1.

In an ideal model, only one specific particle is probed in a single measurement, and the other particles are all implicit ones hiding in the surrounding liquid environment as the medium. This assumption helps to reduce the dressed many-particle problem to a dressed single-particle one, so that the subscripts $i$ distinguishing different particles could be deleted. If there is no external driving force, a self-consistent equation of the particle velocity dynamics could be derived by substituting Eq. S8 into Eq. 3,

$$
\frac{d v}{d t}=-\gamma_{v} \boldsymbol{v}+\boldsymbol{R}_{\boldsymbol{v}}-\iiint \widehat{N}_{(\boldsymbol{x}, \boldsymbol{y})} \tilde{G}\left(\boldsymbol{x}-\boldsymbol{x}^{\prime}, t-t^{\prime}\right) \widehat{M}_{\left(\boldsymbol{x}^{\prime}, \boldsymbol{y}^{\prime}\right)} \boldsymbol{v}\left(t^{\prime}\right) d \boldsymbol{x}^{\prime} d t^{\prime} d \boldsymbol{x}
$$

where $\gamma_{v}$ and $\boldsymbol{R}_{\boldsymbol{v}}$ are the intrinsic damping constant and fluctuation force of the target particle moving in an equilibrium medium, and $\widehat{M}_{\left(x^{\prime}, y^{\prime}\right)}$ denotes the transformed operator $\widehat{M}_{\left(x^{\prime}, y_{i}^{\prime}\right)}^{\prime}$ in a

\begin{tabular}{|c|c|c|}
\hline$\widehat{L}_{\phi}^{\prime}$ & $\widetilde{\boldsymbol{G}}$ & $\phi$ \\
\hline$\frac{\partial}{\partial t}+\gamma$ & $H(t) e^{-\gamma t}$ & Dissipative field \\
\hline$\frac{\partial^{2}}{\partial t^{2}}+2 \gamma \frac{\partial}{\partial t}+\omega_{0}^{2}$ & $\begin{array}{l}H(t) e^{-\gamma t} \frac{\sin \left(\omega^{\prime} t\right)}{\omega^{\prime}} \\
\text { where } \omega^{\prime}=\sqrt{\omega_{0}^{2}-\gamma^{2}}\end{array}$ & Damped oscillating field \\
\hline$\frac{\partial}{\partial t}-D \frac{\partial^{2}}{\partial x^{2}}$ & $H(t)(4 \pi D t)^{-\frac{1}{2}} e^{-\frac{x^{2}}{4 D t}}$ & Diffusive field \\
\hline$\frac{\partial^{2}}{\partial t^{2}}-c^{2} \frac{\partial^{2}}{\partial x^{2}}$ & $\frac{H\left(t-\left|\frac{x}{c}\right|\right)}{2 c}$ & Field with wave-like transportation \\
\hline$\frac{\partial^{2}}{\partial t^{2}}+2 \gamma \frac{\partial}{\partial t}-c^{2} \frac{\partial^{2}}{\partial x^{2}}$ & $\begin{array}{l}\frac{e^{-\gamma t}}{2}\{\delta(c t-x)+\delta(c t+x)+ \\
\left.H(c t-|x|)\left[\frac{\gamma}{c} I_{0}\left(\frac{\gamma u}{c}\right)+\frac{\gamma t}{u} I_{1}\left(\frac{\gamma u}{c}\right)\right]\right\} \\
\text { where } u=\sqrt{c^{2} t^{2}-x^{2}}\end{array}$ & $\begin{array}{l}\text { Field with hyperbolic heat } \\
\text { conduction }\end{array}$ \\
\hline
\end{tabular}
simplified single-particle fashion.

Table S1. Examples of the associated pairs of $\hat{L}_{\phi}^{\prime}$ and the Green's functions. $H(t)$ is the Heaviside step function and $I_{a}(x)$ is the modified Bessel function of the first kind ${ }^{1}$. 


\section{The local-coupling limit of dressed dynamics equation}

In the particle's dressed dynamics, if both $\widehat{M}_{(x, y)}$ and $\widehat{N}_{(x, y)}$ refer spatially local coupling between particle and medium, i.e., $\widehat{M}_{(\boldsymbol{x}, \boldsymbol{y})} \boldsymbol{v}=\delta(\boldsymbol{x}-\boldsymbol{y}) \widehat{M} \boldsymbol{v}$ and $\widehat{N}_{(\boldsymbol{x}, \boldsymbol{y})} \phi=\delta(\boldsymbol{x}-\boldsymbol{y}) \widehat{N} \boldsymbol{\phi}$, where $\widehat{M}$ and $\widehat{N}$ are the space-independent operators as in Eq. 2, and $\delta$ denotes Dirac delta function in space, the particle velocity dynamics has the specific path-correlated form as:

$$
\frac{d v}{d t}=-\gamma_{v} \boldsymbol{v}+\boldsymbol{R}_{\boldsymbol{v}}-\widehat{N} \int \tilde{G}\left(\boldsymbol{y}(t)-\boldsymbol{y}\left(t^{\prime}\right), t-t^{\prime}\right) \widehat{M} \boldsymbol{v}\left(t^{\prime}\right) d t^{\prime}
$$

When the particle is not in the ballistic motion, i.e., $\boldsymbol{y}$ does not increase linearly with time, the last term on the right-hand side of Eq. S10 is not a standard convolution equation as that in the linear response theory ${ }^{2}$, but rather a path-dependent integral. This indicates the spacedependent dressed diffusion model is an extension of previous theory by generalizing the nearequilibrium memory convolution to an integral with both path and time dependences.

\section{An aqueous solvation shell model}

Here we apply spatio-temporal dressed dynamics equation to study an aqueous solvation shell model where hydrogen bond network is present. We first calculate the intrinsic dynamics of the free relaxation of the field $\phi^{R}(x, t)$ when there is no external driving force or coupling from the target particle but with the system boundary, and then seek the corresponding operator $\hat{L}_{\phi}^{\prime}$. The relaxation dynamics of $\phi^{R}(x, t)$ is described by the multi-shell model for a crowded system of finite size, and the explicit form is expressed in Eq. 10. Because Eq. 9 characterizes the intrinsic relaxation process inside the system with the size parameter $l$, so it is the solution of the homogeneous differential equation for the self-relaxation dynamics where the system size effect can be absorbed into the operator itself and no additional function is required to describe the boundary condition. From the expression of $\phi^{R}(x, t)$, we build one corresponding operator $\hat{L}_{x}$ as follows:

$$
\hat{L}_{x}=\frac{\partial^{2}}{\partial t^{2}}+K(x) \frac{\partial}{\partial t}
$$

where $K(x)=k_{1}=\frac{k}{\xi}(l-x)^{\frac{\beta}{2 \rho \Delta r}} \exp \left(-\frac{\lambda}{4 k_{B} T}\right), k$ is the collision frequency associated with shell reaction, and $\xi=\exp \left(\frac{E \Delta r}{2 k_{B} T}\right)$ as introduced in Eq. 8. Based on Eq. S11, it is shown that $t$ is the only variable acted on by $\hat{L}_{x}$, and $x$ can be treated as the parameter. So, its associated Green's function which fulfills the relation $\hat{L}_{x} \widetilde{G}_{x}(t)=\delta(t)$ is

$$
\tilde{G}_{x}(t)=\frac{H(t)}{K(x)}\left[1-e^{-K(x) t}\right]
$$

where $H(t)$ is the Heaviside step function. In this pair of $\hat{L}_{x}$ and $\tilde{G}_{x}$, only time is the related variable. In other words, the inhomogeneous solution $\phi$ of $\hat{L}_{x}$ is solved by $\phi(x, t)=$ $\int \tilde{G}_{x}\left(t-t^{\prime}\right) f_{x}\left(t^{\prime}\right) d t^{\prime}$, where $f$ is the driving function that has both spatial and temporal dependences. However, these are not the only pair of $\widehat{L}_{\phi}$ and $\tilde{G}$. There is no such a unique intrinsic operator for the specific $\phi^{R}(x, t)$ during the free relaxation process, because the free field is the homogeneous solution of $\hat{L}_{\phi}$, i.e., $\hat{L}_{\phi} \phi^{R}(x, t)=0$, so the corresponding $\hat{L}_{\phi}$ form an abelian group with the sum operation where the identify element of this group is the trivial 
zero operator. In the specific case shown in Eq. S11, $\hat{L}_{x}$ describes a space-dependent damped field, which is consistent with the physical insight about the non-equilibrium relaxation dynamics of the shells formed by the non-local bonding clusters.

Combining Eq. S12 with the velocity dynamics of dressed diffusion, we have the selfconsistent velocity dynamics for the dressed dynamics of the tracked particle in the aqueous medium with the presence of hydrogen bond network:

$$
\frac{d v}{d t}=-\gamma_{v} v+R_{v}-\iint \widehat{N}_{(x, y)} \tilde{G}_{x}\left(t-t^{\prime}\right) \widehat{M}_{(x, y)} v\left(t^{\prime}\right) d t^{\prime} d x
$$

Then we assume that the collisional excitation operator $\widehat{M}_{(x, y)}$ and the medium-particle

damping force operator $\widehat{N}_{(x, y)}$ follow $\widehat{M}_{(x, y)}=\frac{\partial}{\partial t^{\prime}}$ and $\widehat{N}_{(x, y)}=C \cdot \delta(x-y(t))$, where $C$ is a constant for the coupling force density normalized with mass. Averaging the stochastic dynamics over non-equilibrium ensembles, the dressed dynamics of the velocityautocorrelation (VAC) function $\left\langle v v_{0}\right\rangle$ is written as follows:

$$
\left[\frac{d}{d t}+\gamma_{v}\right]\left\langle v v_{0}\right\rangle(t)=\left\langle v_{0}^{2}\right\rangle \delta(t)-C \int e^{-K(\bar{y})\left(t-t^{\prime}\right)}\left\langle v v_{0}\right\rangle\left(t^{\prime}\right) d t^{\prime}
$$

where $\delta(t)$ is the Dirac Delta function, $\bar{y}=\sqrt{\left\langle y^{2}\right\rangle(t)},\left\langle y^{2}\right\rangle(t)=\int_{0}^{t} \int_{0}^{t}\left\langle v\left(t_{1}\right) v\left(t_{2}\right)\right\rangle d t_{1} d t_{2}$ is the mean squared displacement (MSD), and $v_{0}$ is the measured velocity at the initial time.

\section{Confinement effect on dressed dynamics of an aqueous solvation shell}

We analyze the size effect of the confined system both numerically and analytically. We assume that the system size varies from several nanometers to around micrometer to characterize the typical size of local domains in a wide range of chemical and biophysical systems $^{3-6}$. When the system size is limited to $2 \mathrm{~nm}$, the long-time motion is different from the case in a larger system. During the first microsecond, the target particle goes a fast relaxation process that guides itself beyond the critical size of the system to the outer strong trapping boundary of long-lasting boundary shell. Then the movement of the particle slows down due to the strong viscoelastic force at the boundary layer. Approaching the long-time limit, the trapping boundary will contribute to a late response force that bounce the particle back inside and due to the stochastic nature of the diffusive motions, the particle's MSD goes back towards a saturation value around the squared system size. This phenomenon only shows when the system size is small enough that the core particle reaches far enough before the intermediate diffusion stage. From the numerical solution, it is clearly illustrated that when the system size changes from large to small, the MSD shows opposite dynamics at the large time scales while preserving the same fast-scale characteristics. In addition, although the long-time tails have different signs, the scaling exponents of the related power-law relations are the same.

\section{Time resolved viscosity of aqueous solvation shell system}

In temporal dressed diffusion model, the damping force acting on the target particle is expressed by an integral term involving spatial-temporal Green's functions, and in the linear region, it could be simplified into a convolution term, in consistence with the linear response 
theory. The generalized damping interaction describes the dynamic evolution of the kinetic state of the probed particle and provides a microscopic perspective for the liquid viscosity, the dynamic property associated with the transport process of liquid system in real space. Following the Stokes-Einstein method, we define viscosity from the dynamic diffusion coefficient:

$$
\eta=\frac{k_{B} T}{6 \pi r_{c} D}
$$

where $r_{c}$ is the radius of the target particle and $D=\left\langle y^{2}\right\rangle / t$ is the time-dependent diffusion coefficient. Since viscosity quantifies the spatial transportation in liquid state, the corresponding timescale is the same as the dressed diffusion region, except the ballistic timescale corresponding with a transient super-diffusion state and the long-time confinement due to the size limitation. As a result, the explicit time-dependent viscosity is shown as:

$$
\eta=\frac{m_{c} t}{12 \pi r_{c}}\left[b_{1}\left(\frac{t}{a_{1}}-\frac{1-e^{-a_{1} t}}{a_{1}^{2}}\right)+b_{2}\left(\frac{t}{a_{2}}-\frac{1-e^{-a_{2} t}}{a_{2}^{2}}\right)\right]^{-1}
$$

where equipartition is assumed for the velocity degree of freedom, $m_{c}$ is the inertial mass of the target particle, and $a_{i}$ and $b_{i}$ are the parameters explained in Eq. 12 and explicitly solved in temporal dressed diffusion model. ${ }^{7}$ There are two critical values of viscosity characterizing two distinct states at separate timescales: the bare viscosity $\eta_{1}$ for the short-lived Langevin state where the active particle-medium interaction has not shown up when $1 / a_{1} \ll t \ll 1 / a_{2}$, $\left(e^{-a_{1} t} \rightarrow 0\right.$ and $\left.e^{-a_{2} t} \rightarrow 1-a_{2} t\right):$

$$
\eta_{1}=\frac{m_{c}}{12 \pi r_{c}} \frac{a_{1}\left(a_{1}-a_{2}\right)}{a_{1}-\gamma_{\bar{\phi}}} \approx \frac{m_{c}}{12 \pi r_{c}} \gamma_{v}=\eta_{0}
$$

where $\eta_{0}$ is the classical viscosity based on Stokes-Einstein relation for Brownian diffusion; and the dressed viscosity $\eta_{2}$ dominated by the slow and non-local response through liquid medium when $t \gg 1 / a_{2},\left(e^{-a_{1} t} \rightarrow 0\right.$ and $\left.e^{-a_{2} t} \rightarrow 0\right)$ :

$$
\eta_{2}=\frac{m_{c}}{12 \pi r_{c}}\left(\gamma_{v}+\frac{\bar{M} \bar{N}}{\gamma_{\bar{\phi}}}\right)=\eta_{1}\left(1+\frac{\bar{M} \bar{N} \xi}{\gamma_{v} k} l^{-\frac{\beta}{2 \rho \Delta r}} e^{\frac{\lambda}{4 k_{B} T}}\right)
$$

where $\bar{M} \bar{N}$ is the product of the effective coupling strengths in Eq. 13. If the characteristic length $l$ is finite and the reorganization energy is higher than $4 k_{B} T$, the second term inside the parenthesis of Eq. S17b is dominant, i.e.,

$$
\eta_{2} \approx \eta_{1} \Gamma e^{\frac{\Delta \bar{G}}{k_{B} T}}
$$

where $\Gamma=\frac{\bar{M} \bar{N}}{\gamma_{v} k}$, and $\Delta \bar{G}$ is the free energy barrier defined in Eq. 7 . 


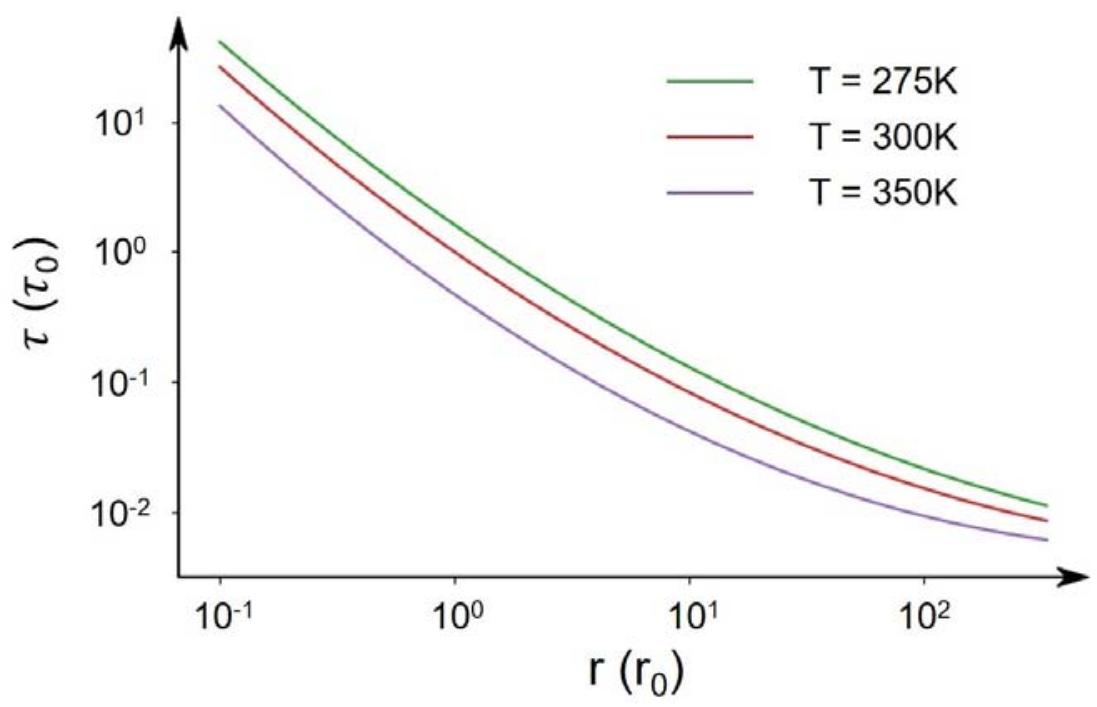

Figure S1. The radius dependence of the lifetime distribution of aqueous solvation shells at different temperatures.

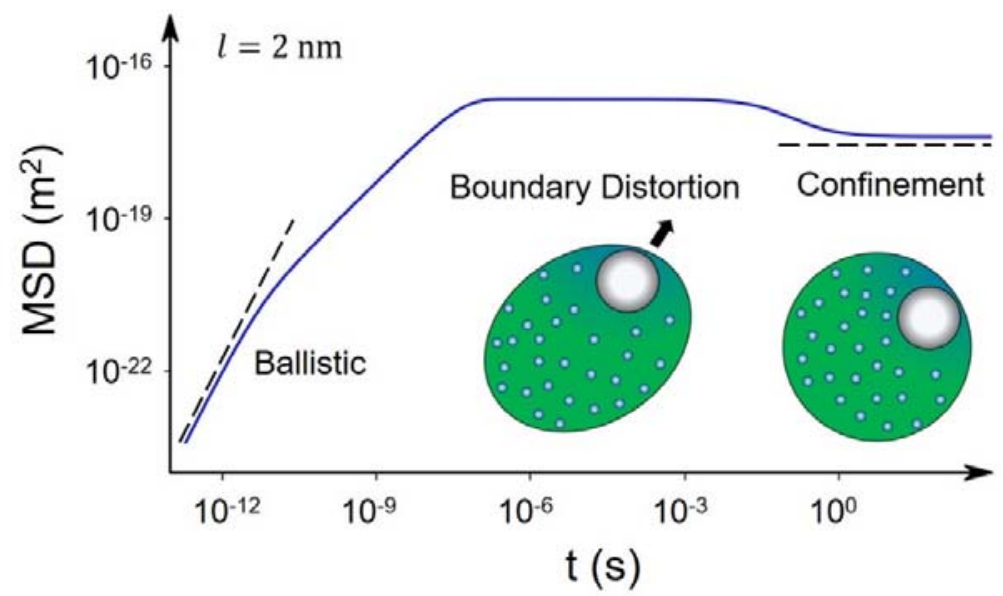

Figure S2. The MSD dynamics of the particle for the system with $l$ of $2 \mathrm{~nm}$. The MSD reaches its maximum value, which is larger than $l^{2}$ at intermediate times, followed by gradual decrease towards the asymptotic limit, $l^{2}$, due to the slow viscoelastic response near the boundary layer. 


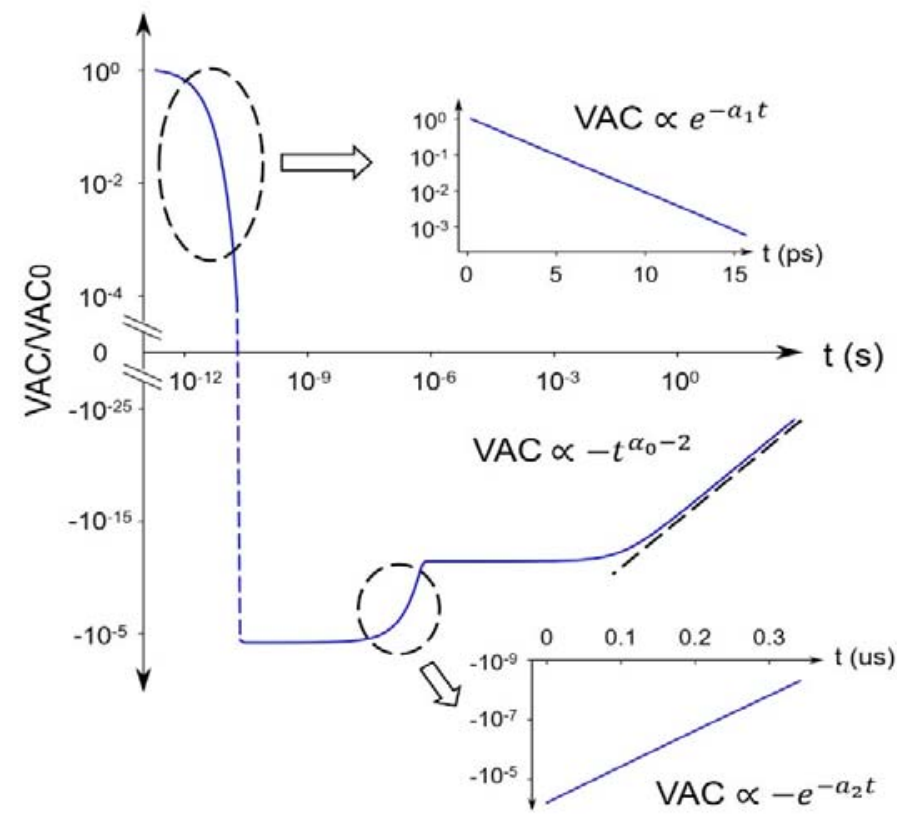

Figure S3. The VAC function of particle for the system with $l$ of $1 \mathrm{um}$. Three different scaling laws are explicitly shown at distinct temporal scales: two exponential laws in pico- and microsecond scales and one negative power-law tail in the confinement limit.

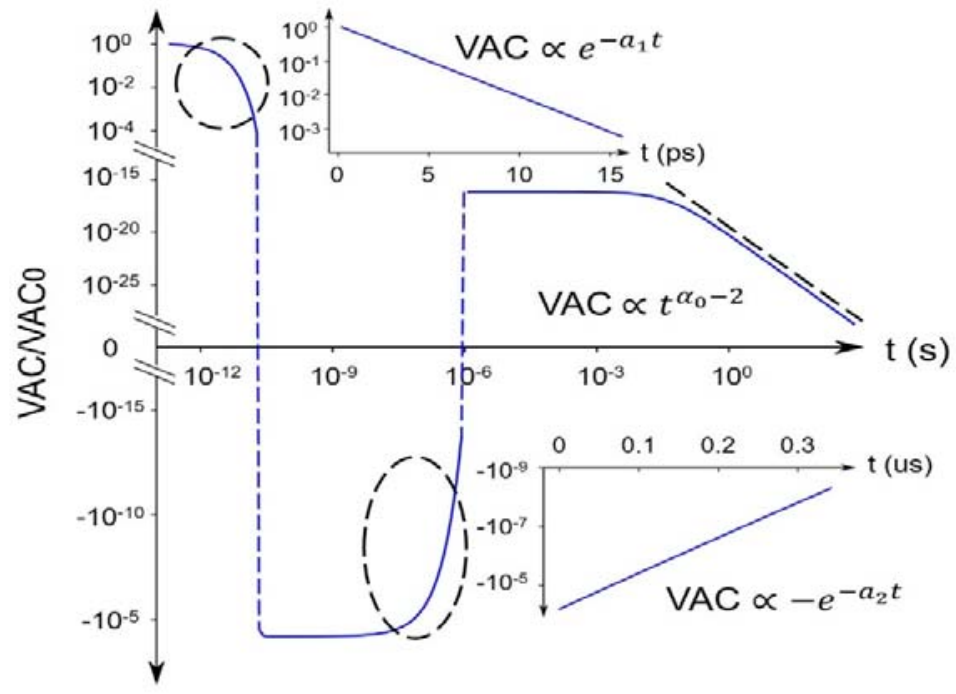

Figure S4. The VAC function of the particle for the system with $l$ of $2 \mathrm{~nm}$. The tail of VAC at the confinement limit has opposite sign in contrast to the VAC for the system with $l$ of 1 um. The power-law exponent remains the same, as shown in the confinement limits at both Figure S3 and S4. 


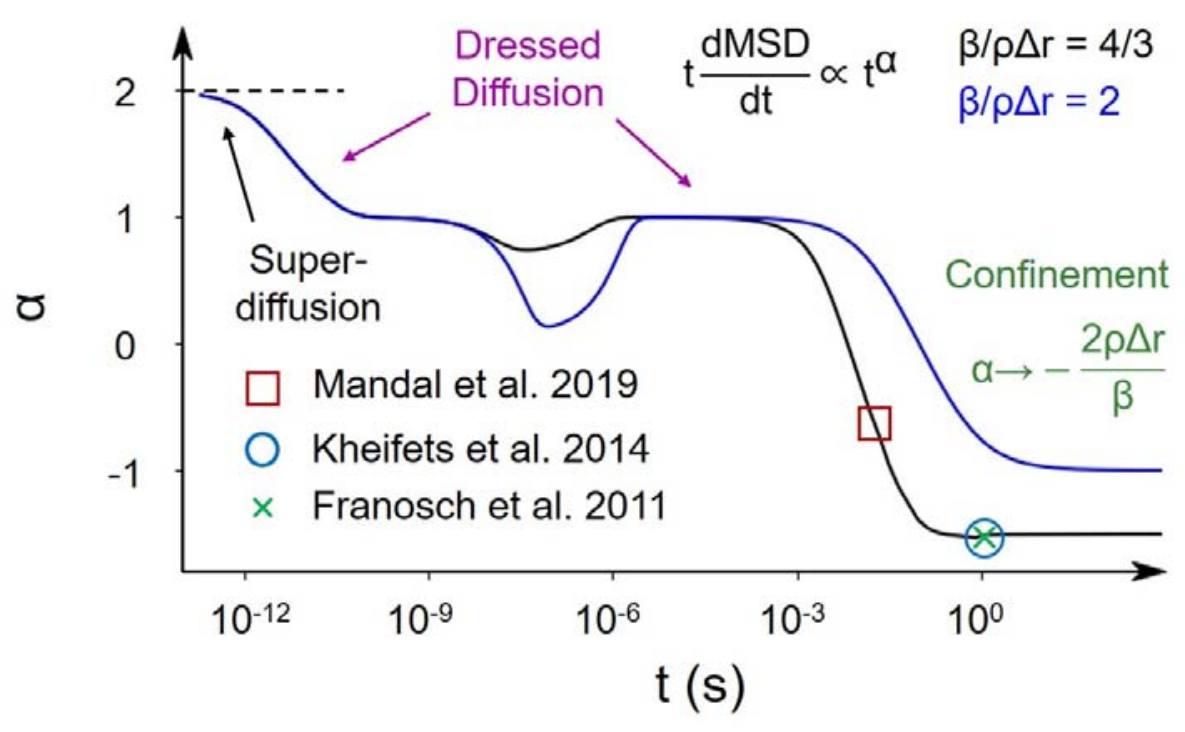

Figure S5. The time-dependent dynamic exponent of the dressed dynamics of aqueous solvation shells. The black curve illustrates the normal ambient water solution with the free parameter fitted with related experimental data at large times. The blue curve denotes the system with more stable solvation shell network. Compared with the dynamic exponent in the water system under ambient condition, the $\alpha$ of solvation shell dynamics in the liquid cell is smaller in the dressed diffusion region, corresponding to a decreased diffusion coefficient consistent with experimental observation in liquid cell electron microscopy, ${ }^{8}$ and is predicted to exhibit larger value at the confinement limit in the present work.

\section{References:}

(1) Duffy, D. G. Green's Functions with Applications, 2nd ed.; Advances in Applied Mathematics; CRC Press: New York, 2015.

(2) Kubo, R. The Fluctuation-Dissipation Theorem. Reports Prog. Phys. 1966, 29 (1), 255-284.

(3) Xue, C.; Zheng, X.; Chen, K.; Tian, Y.; Hu, G. Probing Non-Gaussianity in Confined Diffusion of Nanoparticles. J. Phys. Chem. Lett. 2016, 7 (3), 514-519.

(4) Wang, D.; Wu, H.; Liu, L.; Chen, J.; Schwartz, D. K. Diffusive Escape of a Nanoparticle from a Porous Cavity. Phys. Rev. Lett. 2019, 123 (11), 118002.

(5) Kim, K.-S.; Kim, C.; Karniadakis, G. E.; Lee, E. K.; Kozak, J. J. Density-Dependent Finite System-Size Effects in Equilibrium Molecular Dynamics Estimation of Shear Viscosity: Hydrodynamic and Configurational Study. J. Chem. Phys. 2019, 151 (10), 104101.

(6) Szymański, J.; Patkowski, A.; Wilk, A.; Garstecki, P.; Holyst, R. Diffusion and Viscosity in a Crowded Environment: From Nano- to Macroscale. J. Phys. Chem. B 2006, 110 (51), 25593-25597.

(7) Zhu, K.C.; Su, H.B. Unraveling Dynamic Transitions in Time-Resolved Biomolecular Motions by A Dressed Diffusion Model. J. Phys. Chem. A 2020, 124 (4), 613-617.

(8) Ross, F. M. Opportunities and Challenges in Liquid Cell Electron Microscopy. Science 2015, 350 (6267). 